\title{
UTERINE RUPTURE WITH SIMULTANEOUS BLADDER RUPTURE - A CASE REPORT
}

\author{
S.R. Mudanur ${ }^{1}$, Vaishnavi $A^{2}$
}

\section{HOW TO CITE THIS ARTICLE:}

S.R. Mudanur, Vaishnavi A. "Uterine Rupture with Simultaneous Bladder Rupture - A Case Report". Journal of Evolution of Medical and Dental Sciences 2014; Vol. 3, Issue 02, January 13; Page: 386-389,

DOI: $14260 /$ jemds/2014/1838

\begin{abstract}
Uterine rupture in pregnancy is a rare and often a catastrophic complication with a high risk of fetal and maternal morbidity and mortality. Uterine rupture associated with urinary bladder rupture is a rare but a challenging situation for a practicing obstetrician. A 29 year old $2^{\text {nd }}$ gravida with full term gestation with previous LSCS came with complaints of labor pains and P/V leak of 12 hours. There were features suggestive of rupture uterus with simultaneous bladder rupture. A diagnosis of $\mathrm{G}_{2} \mathrm{P}_{1} \mathrm{D}_{1}$ with term gestation with previous LSCS with rupture uterus with rupture bladder with intrauterine fetal death was made. There was rupture of the previous scar with extension to the anterior vaginal wall with rupture of the posterior wall of the bladder. Uterine and bladder rupture were repaired and peritoneal flap interposed between them and hence conservatively managed. Bladder rupture may be associated with uterine rupture during attempted vaginal birth after cesarean. The potential for bladder injury should be included in the patient's antepartum counseling.
\end{abstract}

KEY WORDS: Rupture uterus, LSCS, labor, vaginal birth after cesarean, bladder rupture.

INTRODUCTION: Uterine rupture in pregnancy is a rare and often a catastrophic complication with a high risk of fetal and maternal morbidity and mortality. Rupture of the uterus can occur in a scarred or an unscarred uterus. The risk of uterine rupture ranges from $0.5 \%$ to $9 \%$ depending upon the type and location of the previous uterine incision. Rupture of the uterus can also involve the other adjacent organs and the most common involvement being the urinary bladder. This being the most common obstetric emergency met in the developing countries.

Here we report a case of $2^{\text {nd }}$ gravida with previous LSCS who presented with rupture uterus with simultaneous rupture of urinary bladder with intrauterine fetal death.

CASE REPORT: A 29 year old $2^{\text {nd }}$ gravida with full term gestation came with complaints of labor pains of $13 \frac{1}{2}$ hours duration and per vaginal bleeding of 12 hours duration. She also complained of vomiting and shoulder pain. She had regular antenatal care which was uneventful. She was G2P1D1 with previous preterm emergency LSCS done for eclampsia, baby died after 1 month due to the complications of preterm birth. Patient was referred in view of non-progress of labor and anemia from peripheral hospital.

On examination patient had a pulse rate of $100 \mathrm{bpm}$ and blood pressure of 100/80 mm of $\mathrm{Hg}$. She appeared pale. Cardiovascular and respiratory system examination was normal. Abdomen was distended, tense and tenderness present. Uterine contour could not be made out and fetal parts were felt easily. On auscultation FHR not heard. On per vaginal examination, cervix was $4 \mathrm{~cm}$ dilated, well effaced and pulled up. Membranes were absent and the presenting part was high up. On catheterization frank hematuria was noted. 
A diagnosis of $\mathrm{G}_{2} \mathrm{P}_{1} \mathrm{D}_{1}$ with full term pregnancy with cephalic presentation with previous LSCS with rupture uterus with IUD was made.

Her investigations revealed:

$\mathrm{Hb} \%-12.9 \mathrm{gm} / \mathrm{dl}$,

Platelet count -2.9 lakh/cumm,

S.creatinine $-1.3 \mathrm{mg} / \mathrm{dl}$,

B.urea $-23 \mathrm{mg} / \mathrm{dl}$,

Blood group and Rh type - B positive,

HIV, HbsAg- negative.

Laparotomy: The patient was taken for emergency laparotomy under general anesthesia. Introperatively hemoperitoneum with blood clots of 500gms were noted. Rupture of previous LSCS scar noted with an extension to the anterior vaginal wall \& tear in the posterior wall of urinary bladder was noted. A still born baby displaced in the peritoneal cavity was extracted. Placenta was completely separated and found in the abdominal cavity. Uterine rupture was managed conservatively with freshening of the edges and sutured with no.1 polyglactin (vicryl) in 2 layers. The urinary bladder was opened at the fundus and rupture in the posterior wall of the bladder was defined clearly. As practiced by the urologists of the institute the bladder rent was closed in 2 layers with no 2-0 polyglactin (vicryl) and peritoneal flap interposed between the bladder and uterus.

DISCUSSION: The clinical presentations of concomitant uterine and bladder rupture at the time of VBAC are variable and depend on the time, location and type of uterine rupture that extends onto the adjacent organs. Acute signs and symptoms can result when a complete rupture occurs intrapartum, including a non-reassuring fetal heart tracing 1,2 , receding of the presenting part on pelvic examination, change in uterine shape, and cessation of uterine contractions, abdominal pain, vaginal bleeding, and even maternal shock ${ }^{3,4}$. Gross hematuria is the most common sign of uterine rupture associated with bladder rupture. Other rare presentations such as vernixuria, meconium stained urine, urinary incontinence induced by vesicouterine fistulas, fever, and urinary tract infections have also been noted ${ }^{5-8}$. The diagnosis requires a high degree of suspicion and awareness.

Various risk factors in relation to uterine rupture during a trial of labor have been identified, including a classic uterine incision, induction with prostaglandins, single-layer closure of a prior uterine incision, an inter pregnancy interval $<18$ months, and a prior preterm cesarean delivery ${ }^{9}$ due to poor wound healing in an undeveloped lower segment of the uterus ${ }^{10}$. Even if the incision is transverse, it would likely encounter the same problems with adequate healing as a classical incision.

In our patient the rupture occurred intrapartum as she had the following risk factors

- Previous pre term LSCS

- Inter pregnancy interval of 18 months (1 $1 \frac{1}{2}$ years)

- Prolonged trial of labor 


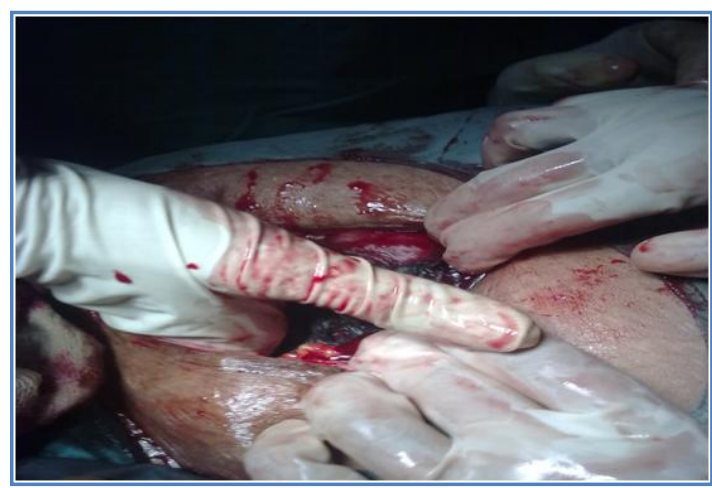

Fig. 1: Baby freely floating in the abdominal cavity

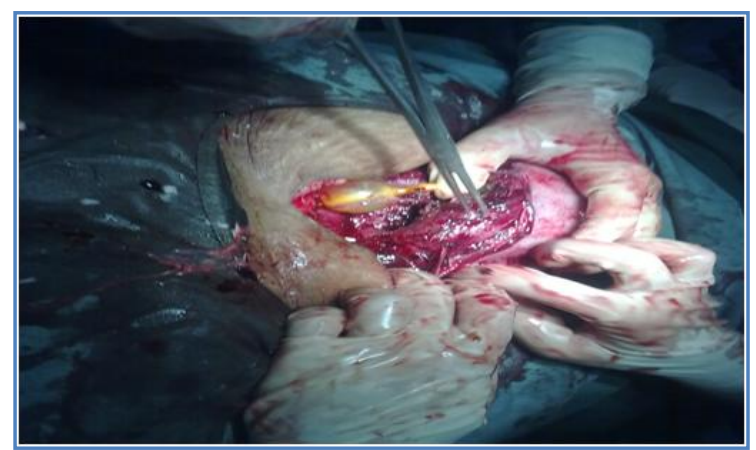

Fig. 2: Foley's bulb visualized through a rent in the posterior wall of the bladder and site of rupture of the scar

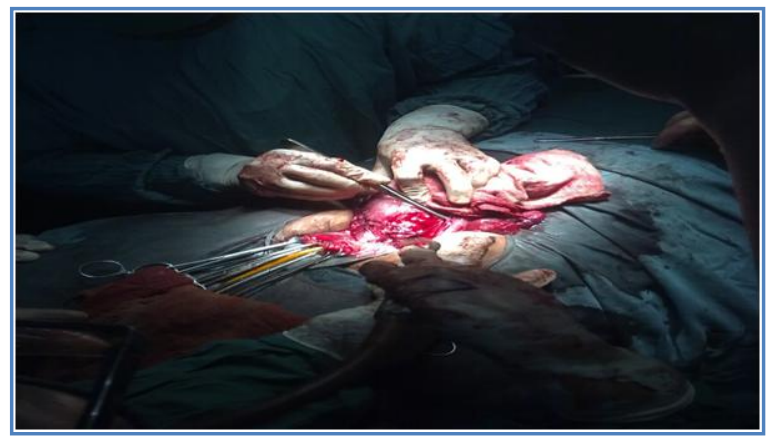

Fig. 3: Repaired uterine rupture and bladder rupture with foley's through the anterior wall of the bladder.

CONCLUSION: The high maternal morbidity, mortality and fetal mortality that follow uterine rupture calls for an integrated effort to prevent its causes. Good ANC, Family planning services, prompt referral of obstructed labor, availability of transportation and obstetric care are the essential factors to prevent uterine rupture and to decrease the maternal mortality, fetal mortality and maternal morbidity associated with it. The case is reported for its rarity and to discuss the treatment modality for optimal results. 


\section{REFERENCES:}

1. Quilligan EJ. Vaginal birth after cesarean section: 270 degrees. J Obstet Gynaecol Res 2001; 27: 169-73.

2. Rageth JC, Juzi C, Grossenbacher H. Delivery after previous cesarean: a risk evaluation. Obstet Gynecol 1999; 93: 332-7.

3. McMahon MJ. Vaginal birth after cesarean. Clin Obstet Gynecol 1998; 41:369-81.

4. Yap OWS, Kim ES, Laros RK. Maternal and neonatal outcomes after uterine rupture in labor. Am J Obstet Gynecol 2001; 184: 1576-81.

5. Kattan SA. Maternal urological injuries associated with vaginal deliveries: change of pattern. Int Urol Nephrol 1997; 29: 155-61.

6. Popli K, Puri M, Gupta A. Cord prolapse though the urethra. Aust N Z J Obstet Gynaecol 2002; 42: 413.

7. O'Grady JP, Prefontaine M, Hoffman DE. Vernixuria: another sign of uterine rupture. J Perinatol 2003; 23: 351-2.

8. Gupta A, Chauhan M, Dahiya P, Sangwan K. Meconium stained urine: an unusual sign of combined uterine and bladder rupture. Aust N Z J Obstet Gynaecol 2005; 45: 334.

9. Smith JG, Mertz HL, Merrill DC. Identifying risk factors for uterine rupture. Clin Perinatol 2008; 35: 85-99.

10. Bergeron ME, Jastrow N, Brassard N, Paris G, Bujold E. Sonography of lower uterine segment thickness and prediction of uterine rupture. Obstet Gynecol 2009; 113: 520-2.

\section{AUTHORS:}

1. S.R. Mudanur

2. Vaishnavi A.

\section{PARTICULARS OF CONTRIBUTORS:}

1. Professor, Department of Obstetrics and Gynaecology, BLDEU's Shri. B.M. Patil Medical College Hospital and Research Centre, Bijapur, Karnataka.

2. Junior Resident, Department of Obstetrics and Gynaecology, BLDEU's Shri. B.M. Patil Medical College Hospital and Research Centre, Bijapur, Karnataka.

\section{NAME ADDRESS EMAIL ID OF THE}

\section{CORRESPONDING AUTHOR:}

Dr. S.R. Mudanur,

Professor, Department of OBG,

Shri. B.M. Patil Medical College Hospital and

Research Centre, Bijapur, Pincode: 586103.

Email -drmudanurs@gmail.com

Date of Submission: 31/08/2013.

Date of Peer Review: 01/09/2013.

Date of Acceptance: 26/12/2013.

Date of Publishing: 09/01/2014. 